\title{
固体的能谱结构与环面 $T^{2}$ 上一个自 治系统的分枝现象
}

\author{
管 克 英 \\ (北京航空学院应用数学教研空)
}

关词橧、环面上闭曲线、基本群、同伦、分枝

人们关于固体能谱的性质的理性认识在很大程度上可以由求解一维周期势场 $V(x)$ 中的 电子的状态问题而获得. 这里假设一维晶格的 (也是 $V(x)$ 的) 周期为 $\omega>0$. 电子的能谱 就由一维定态 Scbrödinger 方程或 Hill 方程决定:

$$
\frac{d^{2} \psi}{d x^{2}}+(\lambda-V(x)) \psi=0,
$$

其中特征值 $\lambda$ 正比于相应定态的电子的能量.

Hill 方程的一般理论已说明电子的能谱结构一一能带与间隙 (或禁带) 是所有周期势场 所具有的共性 ${ }^{[1]}$. 在这里我们证明这种能谱结构也可用二维环面 $T^{2}$ 上的几何性质来说明.

众所周知,方程(1)等价于 Riccati 方程

$$
-\frac{d Z}{d x}=Z^{2}+(\lambda-V(x))
$$

其中

$$
Z(x)=-\psi^{\prime}(x) / \psi(x)
$$

若再令 $Z=\operatorname{tg} \theta$, 就得到 $\theta$ 所应满足的方程

$$
\frac{d \theta}{d x}=\sin ^{2} \theta+(\lambda-V(x)) \cos ^{2} \theta \text {. }
$$

由于 $V(x)$ 是周期为 $\omega$ 的函数,而 $\sin ^{2} \theta$ 与 $\cos ^{2} \theta$ 都是 $\theta$ 的周期为 $x$ 的函数, 所以方程 (4)可以被看成是二维环面 $T^{2}$ 上的一个自治系统. 这一系统在 $T^{2}$ 上的每一条封闭积分轨 线均对应于方程 (2)的一个实的周期解. 利用这一几何解释, 我们得到以下结果:

命唺 1 对应于方程 (2)的实的周期解的 $T^{2}$ 上的一条封闭积分轨线定同伦于 $T^{2}$ 的基 本群 $\pi_{1}\left(T^{2}\right)^{[2]}$ 中以下形式的元素:

(i) $\alpha * \beta^{0}$ ，当且仅当相应的实周期解是有界的;

(ii) $\alpha^{m} * \beta^{n},(m, n) \in \mathscr{N} \times \mathscr{N}$, 当且仅且相应的实周期解是无界的, 其最小周期为 $m \omega$, 并且在区间 $[0, m \omega)$ 内恰有 $n$ 个无穷远点(即解 $Z(x)$ 在该点的值为无穷), 其中 $\alpha$ 与 $\beta$ 是基本群 $\pi_{1}\left(T^{2}\right)$ 的两个生成元, 它们分别相应于 $T^{2}$ 上的一条封闭经线及一条封闭纬线.

命至 2 存在一个分枝点 $\lambda_{0} \in \mathbf{R}$, 使得

(i) 当 $\lambda<\lambda_{0}$ 时, 方程 (2)恰有两个不同的有界实周期解，相应的 $T^{2}$ 上的闭轨同伦于 
$\alpha * \beta^{0}$;

(ii) 当 $\lambda-\lambda_{0}$ 时,方程 (2) 恰有一个有界的实周期解,相应的 $T^{2}$ 上的闭轨同伦干 $\alpha * \beta^{0}$;

(iii) 当 $\lambda>\lambda_{0}$ 时,方程(2)不存在有界的实周期解，它的任一实解在 $x \rightarrow-\alpha$ 时或在 $x \rightarrow+\infty$ 时均无限次地通过无穷远.

命的3 对于任意自然数对 $(m, n) \in \mathscr{N} \times \mathscr{N}$, 在区间 $\left(\lambda_{0},+\infty\right)$ 内存在分枝点 $\lambda_{s}(m, n)$ 与 $\lambda_{G}(m, n)$, 使得

(i) $\lambda_{s}(1, n) \leqslant \lambda_{G}(1, n), \forall n \in \mathscr{N}$,

$$
\lambda_{s}(m, n)=\lambda_{G}(m, n), \forall m>1, n \in \mathscr{N},
$$

而且当且仅当 $\lambda$ 满足

$$
\lambda_{s}(m, n) \leqslant \lambda \leqslant \lambda_{G}(m, n)
$$

时方程 (2) 存在无界的、实的、周期为 $m \omega$ 的解, 它在 $[0, m \omega)$ 内恰有 $n$ 个无穷远点, 其相应于 $T^{2}$ 上的闭轨同伦于 $\alpha^{m} * \beta^{n}$;

(ii) 若 $n / m<n^{\prime} / m^{\prime}$, 定有 $\lambda_{G}(m, n)<\lambda_{s}\left(m^{\prime}, n^{\prime}\right)$;

(iii) 若 $n / m=n^{\prime} / m^{\prime}$, 定有 $\lambda_{s}(m, n)-\lambda_{s}\left(m^{\prime}, n^{\prime}\right), \lambda_{G}(m, n)=\lambda_{s}\left(m^{\prime}, n^{\prime}\right)$;

(iv) 若对某 $n \in \mathscr{N}$ 有 $\lambda_{s}(1, n)<\lambda_{G}(1, n)$, 可令 $D_{n}$ 表示区间 $\left(\lambda_{s}(1, n), \lambda_{G}(1, n)\right)$, 这时对任意 $\lambda \in D_{n}$ 方程 (2)均恰有两个不同的无界的、实的、周期为 $\omega$ 的解,他在 $T^{2}$ 上的相 应的闭轨均同伦于 $\alpha * \beta^{n}$, 而当 $\lambda=\lambda_{s}(1, n)$ 或 $\lambda=\lambda_{G}(1, n)$ 时方程(2)则仅恰有一个无 界的实的 $\omega$ 周期解, 其相应的 $T^{2}$ 上的闭轨同伦于 $\alpha * \beta^{n}$;

(v) 若对某个 $n \in \mathscr{N}$ 有 $\lambda_{s}(1, n)-\lambda_{G}(1, n)$, 则令 $D_{n}$ 为空集, 这时对于 $\lambda=\lambda_{s}(1, n)=$ $\lambda_{c}(1, n)$ 方程 (2) 的所有的实解均是 $\omega$ 周期的, 它们在 $T^{2}$ 上的相应的闭轨均同伦于 $\alpha * \beta^{n}$;

(vi) 对于 $(m, n) \in \mathscr{N} \times \mathscr{N}, m>1$, 当 $\lambda=\lambda_{s}(m, n)=\lambda_{G}(m, n)$ 时,方程(2)的任 一实解均是 $m \omega$ 周期的, 其相应于 $T^{2}$ 上的闭轨同伦于 $\alpha^{m} * \beta^{n}$.

下面我们用 $\sum$ 表示参数 $\lambda$ 的所有上述的分枝点的集合,即

$$
\Sigma \equiv\left\{\lambda_{0}, \lambda_{s}(m, n), \lambda_{G}(m, n) \mid(m, n) \in \mathscr{N} \times \mathscr{N}\right\},
$$

并且令

$$
\begin{aligned}
& D_{0} \equiv\left(-\infty, \lambda_{0}\right), \\
& D \equiv D_{0} \cup\left[\bigcup_{n \in \mathcal{N}} D_{n}\right], \\
& S=\mathbf{R} \backslash D .
\end{aligned}
$$

命 54 集合 $s$ 恰等于 $\Sigma$ 的闭包, 即 $s-\Sigma$.

命的 5 开集 $D$ 中的开区间对应着方程 (1)的能谱中的间隙，闭集 $S$ 中的闭区间形成了 能谱中的能带.

以上的结果表明固体的能谱结构的确可用二维环面 $T^{2}$ 上有关的自治系统的分枝现象来 解释. 有关结果的严格的数学证明可见文献 [3].

\section{考文嗝}

[1] Eastham, M. S. P., The Spectral Theory of Periodic Differential Fquations, Scottish Academic Press, 1973.

[2] Wilhelm Klingenberg, A Course in Diffenential Geometry, Springer-Verlag, New York, 1978.

[3] Guan Ke-ying, Bifurcation of the periodic differentisl equations with reqularisable infinity, Proc. Roy. Soc. Edin (待发表). 